\title{
Apps como una posibilidad más de comunicación entre la marca y su público: un análisis basado en la valoración de los usuarios
}

\author{
Zahaira GONZALEZ ROMO ${ }^{1}$ \\ Ruth S. CONTRERAS ESPINOSA ${ }^{2}$
}

Universidad de Vic

\begin{abstract}
RESUMEN:
La situación actual del mercado y del sector de las nuevas tecnologías genera la necesidad de que las marcas y las empresas, se comuniquen como entes sociales y a través de medios como las aplicaciones móviles. El presente artículo presenta el análisis de 24 marcas que cuentan con aplicaciones móviles (apps). Se ha tomado en cuenta para ello, la valoración que los usuarios dan a las aplicaciones. Específicamente el estudio se centra en herramientas destinadas al dispositivo iPhone. Las apps, representan a las marcas la posibilidad de contar con un soporte más para comunicarse con sus públicos.
\end{abstract}

PALABRAS CLAVE: Marca; apps; iPhone; imagen; valor de marca

TITLE: Apps as an additonal possibility for the communication between brand and his audience: An analysis based on the users rating

\begin{abstract}
:
The actual situation in the new technology market and sector generates the need that companies and also brands communicate like social entities (social communities) via new tools like app's (applications) for mobile phones. This article presents (outlines) the results of a research that is built of a detailed analyze of 24 brands that are using apps as a tool. In particular the study was focused only on iPhone devices. The unique selection criterion was how the users rated the specific application. For companies and brands apps are an additional opportunity to get in contact (in touch) with their customers.
\end{abstract}

KEY WORDS: Brand; apps; iPhone; image; brand value

\section{Introducción}

En aspectos de comunicación de imagen, Capriotti (2009) explica que las empresas cada día deben plantearse ir más allá, adaptándose a los cambios, entrando incluso en una esfera social. La situación actual del mercado y del sector de las nuevas tecnologías genera la necesidad de que las marcas y las empresas, se comuniquen como entes sociales. Bajo esta premisa, no solo cambia la forma en como la marca se posiciona

1 Dra. en Publicidad y Relaciones Públicas por la UAB. Profesora de Campañas Globales de Comunicación. zahairaf.gonzalez@uvic.cat

2 Dra. en ingeniería Multimedia por la UPC. Profesora en Comunicación digital. ruth.contreras@uvic.cat 
sino que además consigue, gestionada correctamente, generar una actitud favorable en sus públicos.

La gestión estratégica de la marca a través de los medios de comunicación y específicamente a través de las nuevas tecnologías de la comunicación cobra día a día una vital importancia y prácticamente todas las empresas que se precien de llevar una comunicación acorde a sus públicos han desarrollado herramientas y adaptado su comunicación de marca a los diferentes soportes, medios y entornos que emergen en el mercado. Es una cuestión de supervivencia y una metamorfosis que no todas las empresas han conseguido conseguir. «No es la especie más fuerte la que sobrevive, sino la que mejor responde al cambio» ${ }^{3}$.

El desarrollo de la Web 2.0 ha generado un cambio en el paradigma tradicional unilateral, donde el modelo one to many se ve sustituido por un espacio many to many, que permite al público compartir y opinar sobre las marcas, además de comunicarse con ellas a través de plataformas como twitter, facebook o you tube. «Podemos decir que las marcas se sienten cómodas en un entorno virtual atravesado por flujos de información: las marcas no son más que un puñado de valores que circulan en la imaginación de una sociedad» (Scolari, 2008).

Las marcas adoptan la personalidad de sus líderes o de las personas que trabajan en ellas, se acercan a las personas. Esto unido al hecho de que «los dispositivos GPS son cada vez más baratos e integrados a los teléfonos móviles y las herramientas de la Web geográficas ${ }^{4}$ hacen prácticamente ilimitado y casi personalizado el servicio de la marca a sus clientes. Las marcas adaptan su visibilidad al entorno de la comunicación móvil, a los nuevos dispositivos, y aplicaciones para esos dispositivos, que repercuten en la imagen de la marca y por tanto, en la mente de los consumidores.

Tal es el caso de las aplicaciones para smart phones, que se han desarrollado con una virulencia impresionante y que continúa creciendo. La evolución constante de dispositivos cada vez más potentes tecnológicamente y además económicos, impulsan al crecimiento significativo para el uso de estas aplicaciones que no necesariamente son incluidas por el fabricante. En Estados Unidos, eMarketer predice que el número de usuarios móviles que acceden a Internet «pasará de 73.7 millones en 2009 hasta 134,3 millones en el año 2013 $»^{5}$, con estas perspectivas es de suponer que el uso de las aplicaciones para dispositivos móviles, jugará un papel trascendente en el conocimiento y comunicación de la marca.

Las aplicaciones generadas para dispositivos móviles representan a las marcas la posibilidad de contar con un soporte más para comunicarse con sus públicos, y en este

\footnotetext{
Frase de Charles Darwin.

4 DANS, Enrique (2010): Todo va a cambiar. Tecnología y evolución: adaptarse o desaparecer, Barcelona, Grupo Planeta..

5 ElKIN, Noha (2010): «Mobile Marketing Trends, Insights and Best Practices», eMarketer Digital Intelligence.The eMarketer Blog. New York: Investigación y análisis de medios, 26 agosto 2010, Publicación seriada mensual. Disponible en: http://www.emarketer.com/blog/index.php/emarketer-webinarmobile-marketing-trends-insights-practices/ Visitado el 12 de octubre 2010.
} 
artículo se realiza una aproximación al tema mediante un estudio cualitativo exploratorio. Se analizan 24 marcas que cuentan con aplicaciones móviles, y para ello se ha tomado en cuenta la valoración y comentarios que los usuarios realizan a cada una de las aplicaciones, entre otras cosas. El artículo se encuentra dividido en 5 secciones. $\mathrm{La}$ primera sección habla de la importancia de la gestión de marca, la número dos explica que son las aplicaciones móviles y la tercera sección describe el planteamineto de este estudio. La sección cuatro y cinco realizan la descripción de la metodología implementada y los resultados obtenidos junto a una discusión, respectivamente. Finalizamos con las conclusiones sobre el tema.

\section{La importancia de la gestión de marca}

Según Scolari (2008), la marca - entendida como un dispositivo semiótico capaz de producir un discurso- expresa valores y se presenta como un «contrato de interpretación» entre las empresas y los consumidores: la marca propone una serie de valores y los consumidores aceptan, o no, formar parte de ese mundo ${ }^{6}$.

La base primordial según Capriotti (2009), en un entorno saturado donde existe una casi nula diferenciación entre los productos, es la determinación de un conjunto de objetivos que marquen una línea a seguir en el trabajo comunicativo. Es prioritario saber llegar a los públicos a través de las correctas herramientas de comunicación, por eso una correcta gestión de marca permitirá entre otras cosas:

1. Conseguir un posicionamiento en la mente de sus públicos, éste «espacio» en la mente del público es indispensable para poder existir, y se alimenta constantemente de lo que la empresa o la marca dice sobre sí misma y los caminos que utiliza para hacerlo.

2. Facilita su diferenciación con la competencia, pues el solo hecho de existir no garantiza ser recordado o conseguir un posicionamiento, la correcta gestión de la comunicación, permite un uso adecuado de los medios que ayudan a la marca no solo a estar presente, sino a diferenciarse de sus competidores.

3. Conseguir evadir en cierta forma la influencia de factores externos en la decisión de compra. Esto quiere decir que esta imagen construye un esquema de referencia previo sobre el cual pueden reforzar sus decisiones de compra.

«Gracias a la correcta gestión de la imagen, la marca no solo consigue, como ya vimos, la diferenciación, el posicionamiento y la influencia en la decisión de compra, sino que además ayuda a la marca a vender mejor, atraer más inversores e incluso mejores trabajadores» ${ }^{7}$.

6 SCOLARI, Carlos (2008): «Online brands: Branding, possible worlds, and interactive grammars» Semiotica, 169-178

7 CAPRIOTTI, Paul (2009): Branding Corporativo. Fundamentos para la gestión estratégica de la identidad Corporativa, Santiago de Chile, Andros Impresores, Colección Libros de Empresa 


\section{El mercado de las Aplicaciones}

Un dispositivo móvil como el iPhone, «no es solo un soporte de transmisión si no un medio con nuevos formatos, sistemas de financiación y un nuevo lenguaje derivado del tamaño de las pantallas y del carácter interactivo del terminal» ${ }^{8}$. A pesar de que existen otros dispositivos con pantalla táctil y teclado virtual, Apple ha logrado diferenciarse y hacerse popular en el mercado de la tecnología móvil. Durante el año 2007, la empresa de la manzana tomó el $28 \%$ del mercado de dispositivos móviles en EE.UU y en 2008, con la llegada del iPhone $3 G$ se posicionó en el segundo lugar de ventas de dispositivos móviles inteligentes ${ }^{9}$, convirtiéndose rápidamente en el líder del mercado. Hace unos meses la página de Apple Inc puso de relieve la cantidad de descargas realizadas en la tienda: 10 billones de apps ${ }^{10}$. El volumen de descarga en los países asiáticos creció significativamente en los últimos seis meses, pero la proporción de descargas de pago y los ingresos globales siguen a la zaga de los EE.UU (Distimo, 2011).

La App Store de Apple para iPhone continúa siendo el almacén más grande de todas las apps disponibles en el mercado por delante de las tiendas de Android, Nokia y Blackberry (Figura 1). En el mes de marzo la tienda para iPad creció un 12\% a 75.755 solicitudes, de las cuales 34.120 están siendo destinadas solo a iPad, el resto de apps son utilizadas también para iphone, así lo indica el reporte de la empresa Holandesa Distimo $^{11}$. La compañía prevé un crecimiento para el mercado de Android y BlackBerry, siendo esta ultima la que duplicara su tamaño en un período de seis meses y con un crecimiento mayor que la tienda de Nokia.

Otros estudios anuales bajo el nombre de Apps Playbook, suministran información sobre el ecosistema de las apps. The Nielsen Company (2010), menciona en este informe que la mayoría de las aplicaciones son encontradas por los usuarios a través de la búsqueda, y se opta por descargar aplicaciones gratuitas o recomendaciones de amigos y familiares. Las valoraciones y comentarios de otros usuarios son un punto importante cuando se trata de decidir qué aplicación se descargará. Los usuarios del iPhone comentan además, estar dispuestos a pagar por una de cada tres aplicaciones que se descargan, mientras que los usuarios de Android y Blackberry están menos dispuestos a pagar por las aplicaciones. Suelen además seguir las tendencias a la hora de descargar aplicaciones, y esto probablemente tiene que ver con las diferentes opciones que App Store proporciona en comparación con el Android Market, por ejemplo.

VACAS AgUILAR, Francisco (2007): «Telefonía Móvil: la cuarta ventana», ZER, vol. 23,2007, 199-217

CANAlys. Expert Analysis for High-tech Industry: Global Smart Phone Shipments Rise 28\%». Reading. UK (2008). Disponible en: http://www.canalys.com/pr/2008/r2008112.html Visitado el 10 de octubre 2010.

10 Concurso desarrollado por Apple para llegar a la descarga número 10 billones. Finalizada en febrero de 2011. Ver: http://www.apple.com/itunes/10-billion-app-countdown/

11 DISTIMO Report - The battle for the most content and the emerging tablet market. Marzo 2011 
Fig. 1. Número de apps disponibles, basado en Distimo

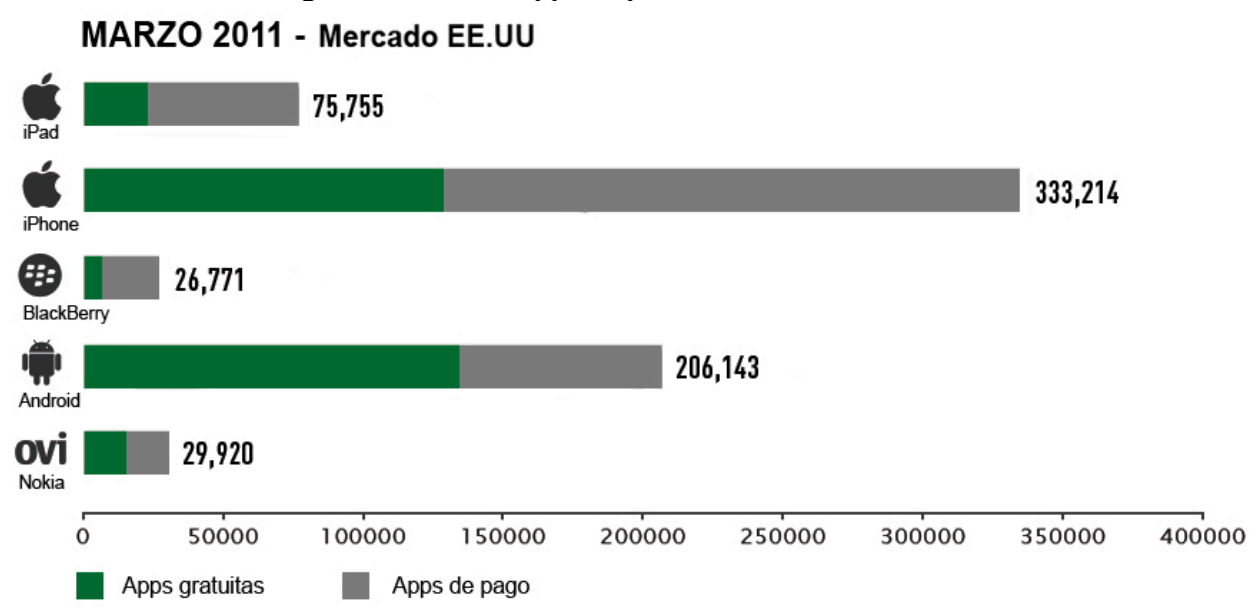

En el ecosistema español, conviven en la actualidad dos concepciones: por un lado la de una plataforma para la comercialización de contenidos meramente adaptados de otros formatos y de otro, la de un medio emergente que hace posible el diseño de contenidos que aprovechen sus especificidades (Feijóo y Maghiros, 2008; Aguado y Martínez, 2009). Pero las tendencias del mercado español no son aún relevantes para los desarrolladores que quieren hacer crecer su negocio.

Eso lo demuestra el informe European App Store Market Report, de Distimo y Mobile Entertainment Forum $(M E F)^{12}$ que menciona el panorama del mercado en Europa, comparando las tiendas de Apple en el Reino Unido, Francia, Alemania, Italia, Polonia, Rusia y España. En la figura 2, se puede observar que más de un tercio de las descargas pertenecen al Reino Unido. Aunque en el estudio sólo se tuvieron en cuenta las 300 principales y más populares apps de pago y gratuitas, el Reino Unido sigue siendo el mercado europeo más importante para los desarrolladores, quedando España situado en un quinto lugar con un 7\% de descargas para iPhone y 9\% para iPad.

12 MEF es la asociación global de la industria de los medios móviles. 
Fig. 2. Descargas de apps en Europa, basado en Distimo

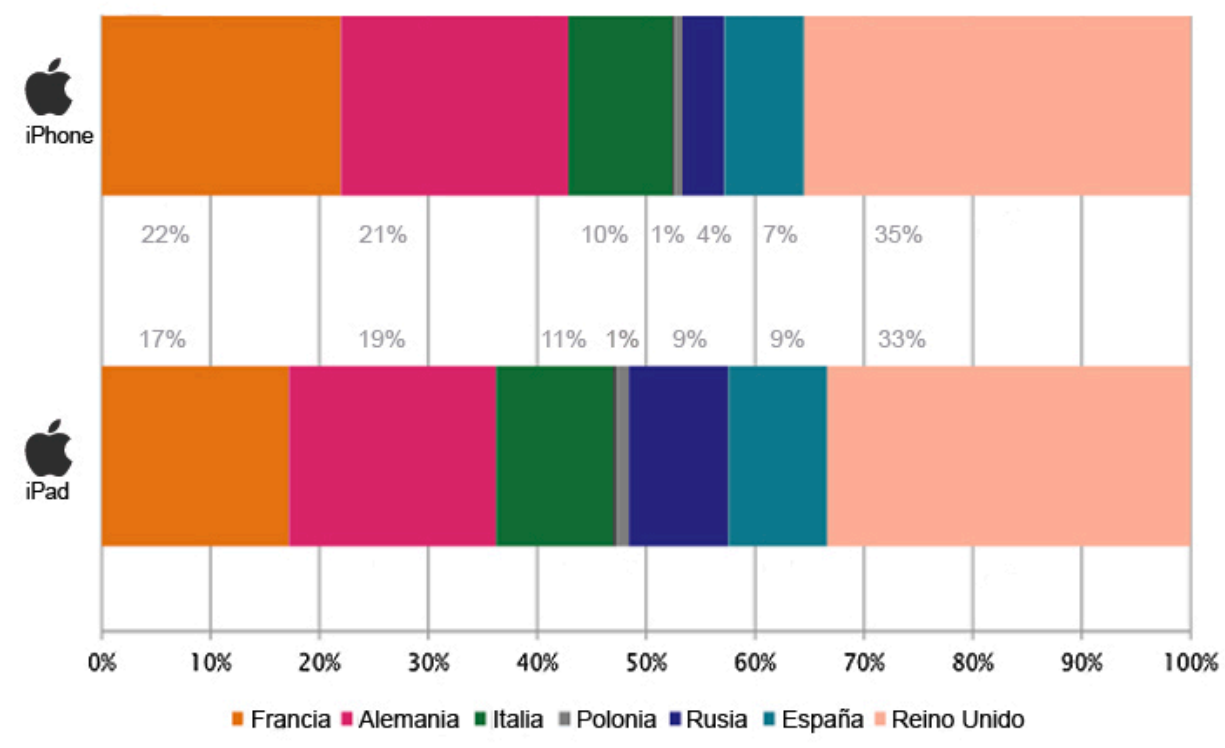

\section{El Planteamiento del estudio}

Para llevar a cabo este análisis es necesario tener en cuenta además de lo que significa y representa la imagen para la marca, las circunstancias actuales del mercado, por ello, los resultados del informe Mobile Marketing Trends, Insights and Best Practices llevado a cabo por Noha Elkin para eMarketer, revista de investigaciones de mercado vinculadas con el mundo digital, cobra especial importancia, pues en este estudio habla directamente sobre el uso de las aplicaciones para la gestión de marca.

Especifica que los anunciantes, han tenido que entender que han de ser accesibles a los consumidores en el móvil, aunque muchos aún no saben como hacerlo. Es indispensable que las marcas estén en donde están sus clientes, tanto desde los medios de comunicación como desde sus dispositivos móviles, por lo que han de considerar éstos como parte del mix en sus estrategias de marketing.

El siguiente paso en este nuevo espacio de comunicación bilateral entre la marca y el cliente es la elaboración de una estrategia coherente y garantizar al usuario una experiencia complementaria y consistente con la marca. En otras palabras, la aplicación no ha de ser un ente separado de la imagen general de la marca y debe ser acorde a la gestión de la misma en las otras herramientas de comunicación que desarrolle. Elkin (2010) destaca tres puntos clave en la gestión de marca para las aplicaciones móviles:

El primero y más importante para elaborar una estrategia coherente, es que los anunciantes deben centrarse en el análisis de las necesidades de los usuarios, determi- 
nar sus objetivos, elaborar planes de integración y, sobre todo, la preparación de los medios de comunicación multicanal y de apoyo a la comercialización.

En segundo lugar, la experiencia del usuario en las aplicaciones móviles debe ser complementaria y coherente con su experiencia a través de otros canales. Cualquier aplicación móvil de marca debe ofrecer una experiencia única, sin embargo, esta experiencia debe mantenerse fiel a la marca y a la identidad de la empresa.

Por último, y como tercer punto destacable, la creación de aplicaciones como extensión de la marca, lo que significa evitar una rápida incorporación a este medio, en favor de la creación de valor, es decir aplicaciones que apoyen la forma en que los públicos ven la marca y que sean coherentes con su estrategia.

El informe de eMarketer señala además, que la experimentación es recomendable, y que incluso, al ser algo mutable y modificable, puede ir creciendo y cambiando en función de la experimentación de la marca. Compara las aplicaciones con, por ejemplo, los catálogos de productos de las tiendas, estos cambian cada temporada y siguen siendo coherentes con la marca.

Significa por tanto, que el desarrollo de una aplicación para móviles debe formar parte de un plan integral de comunicación y gestión de la marca, ya que de otra forma, garantiza un fracaso seguro.

Es obvio que el desarrollo de estas aplicaciones ha de tomar en cuenta de forma indispensable la comprensión del comportamiento del usuario, su intención y aspiraciones y los puntos fuertes y débiles de una plataforma de aplicación dada. Debido a la velocidad de nuestra sociedad conectada, una aplicación mal ejecutada que genera comentarios negativos puede hacer un daño a la marca.

Aunque las apps dispuestas en Apple Store pueden llegar a ser de las herramientas más destacadas debido a su novedad, esta tienda no es el único camino para llegar a los consumidores. Es indispensable recordar que existen además, otras plataformas que siguen encontrando eco en los consumidores y usuarios, como páginas web, blogs y fórums. Lo que lleva a la conclusión de que así como no hay un dispositivo para todos, ninguna plataforma es la más adecuada para todas las marcas.

\section{Metodología}

Para este estudio cualitativo exploratorio, el primer objetivo fue identificar las fuentes de información secundaria que permitieran recoger directamente información del sector.

Para ello, se seleccionó una muestra de aplicaciones para iPhone proporcionadas por Apple Store España, bajo la clasificación «Top gratuitas». Nos hemos enfocado a examinar aplicaciones de Apple, debido a su éxito sobre Android y Blackberry, y nos hemos basado en el informe de The Nielsen Company para seleccionar el grupo de aplicaciones gratuitas, debido a su gran éxito de descarga. 
La muestra analizada se compone de aplicaciones correspondientes a 24 marcas internacionalmente reconocidas y clasificadas en la tienda bajo el concepto; Estilos de Vida. Al ser este un estudio exploratorio, se analizaron las 28 apps enmarcadas en los primeros sitios de popularidad y descarga. El tamaño de muestra reducido se consideró además por los siguientes motivos (Fernández, 2010):

-En estudios exploratorios se utilizan muestras reducidas porque son acciones previas que ayudan a diseñar investigaciones posteriores con muestras más extensas.

-En metodología cualitativa no es necesario utilizar muestras elevadas porque la riqueza de la información resultante depende principalmente de la profundidad del análisis y no de la extensión de la muestra.

-El análisis de resultados no plantea la extrapolación de la información obtenida al universo objeto de estudio. Los resultados obtenidos pueden ser tratados como hipótesis que se podrán contrastar con estudios cuantitativos posteriores con muestras elevadas.

- Cumplen una función de estimulación intelectual preliminar (Corbeta, 2007).

Cabe mencionar además que no todas las aplicaciones que aparecen bajo el concepto «Estilos de Vida» en la App Store como opción de descarga, corresponden a marcas reconocidas. La mayoría de aplicaciones que facilita la tienda, son creadas para la gestión de acciones cotidianas pero no se vinculan específicamente a una marca específica.

Las 24 marcas agrupadas bajo este concepto son; Elle, Tous, eBay, Vogue, Cosmopolitan, Philadelphia, H\&M, Blanco, Uterqüe, Zippo, Mens Health, Telepizza, Nespresso, Mango, Zara Home, D\&G, Gucci, Ikea, La maquinista, Zara, Massimo Dutti, Cinesa, Marie Claire y Glamour.

Para conocer los valores que la aplicación puede aportar a la marca y, en su defecto, considerar si este tipo de aplicación consigue su objetivo a través de la herramienta en función, se ha realizado una clasificación para facilitar el análisis. La clasificación sugerida es: Aplicaciones destinadas a la moda, Aplicaciones para revistas, Aplicaciones destinadas a alimentación, Aplicaciones relacionadas con el hogar y Aplicaciones sobre otros.

El proceso de análisis se basó en el cruce de datos para poder trazar descripciones transversales. Se ha tomado en cuenta: La app desarrollada, la marca que la representa, la categoría de la aplicación, la valoración otorgada por los usuarios en cada caso y las opiniones y comentarios de los usuarios. La valoración dada por los usuarios se refleja en forma de «estrellas». Estas representan una estimación otorgada en función del uso, utilidad y éxito de la aplicación.

Este proceso de información se complementó con un estudio de fuentes bibliográficas que muestran las observaciones de teóricos, directores de marketing y reportes sobre el mercado móvil. Cabe mencionar, que lejos de buscarse la representatividad estadística de los resultados, se trata de ordenar y hacer visible un ámbito poco explorado hasta el momento por la comunidad académica. 


\section{Resultados y discusión}

La Tabla I, muestra las apps y marcas analizadas. Como se describió con anterioridad, las aplicaciones han sido organizadas bajo los criterios de búsqueda Estilos de Vida $>$ Top gratuitas. Apple Store, las clasifica en diferentes categorías, las cuales se describen en la columna número dos. La tabla cuenta además con la valoración en «estrellas» que van de cero a cinco y es asignada por los usuarios, el número de opiniones recibidas por cada app y finalmente la columna número cinco, proporciona una valoración general otorgada por los autores tomando en cuenta la opinión de Jami Lawrence $(2003)^{13}$, quien menciona que es imprescindible que las aplicaciones proporcionen al usuario cualquiera de estas experiencias: diversión, ahorro de tiempo y/o comodidad en la utilización. Teniendo en cuenta estos atributos, si la aplicación cumple con proporcionar una de estas experiencias, es valorada con un 1 punto como deficiente, si cumple con dos de ellos, es valorada con 2 puntos como aceptable, y si cumple con las tres experiencias es valorada con 3 como óptima.

Tabla 1. Valoración de APPs

\begin{tabular}{llrrr}
\hline APP analizada & Categoría & $\begin{array}{l}\text { Valoración } \\
\text { estrellas* }\end{array}$ & $\begin{array}{l}\text { Opiniones } \\
\text { Usuarios }\end{array}$ & $\begin{array}{l}\text { Valoración } \\
\text { General** }\end{array}$ \\
\hline ELLE GOURMET & REVISTAS & 4 & 19 & 3 \\
TOUS & MODA & 3,5 & 129 & 3 \\
EBAY & OTROS & 3,5 & 131 & 3 \\
VOGUE & REVISTAS & 3,5 & 37 & 1 \\
COSMOPOLITAN & REVISTAS & 3,5 & 545 & 1 \\
PHILADELPHIA & ALIMENTACIÓN & 3 & 259 & 2 \\
H\&M & MODA & 3 & 21 & 1 \\
BLANCO & MODA & 3 & 42 & 1 \\
UTERQÜE & MODA & 3 & 16 & 1 \\
ZIPPO & OTROS & 3 & 3035 & 1 \\
MENS HEALTH & REVISTAS & 3 & 56 & 1 \\
COSMOPOLITAN 2.0 & REVISTAS & 3 & 24 & 2 \\
TELEPIZZA & ALIMENTACIÓN & 2,5 & 759 & 1 \\
NESPRESSO & ALIMENTACIÓN & 2,5 & 277 & 1 \\
MANGO & MODA & 2,5 & 112 & 1 \\
ZARA HOME & HOGAR & 2,5 & 140 & 1 \\
D\&G & MODA & 2,5 & 187 & 1 \\
GUCCI & MODA & 2,5 & 338 & 2 \\
\hline
\end{tabular}

13 LawrenCE, Jami (2010): «Best Practices: Mobile Marketing \& App Strategies for Food Brands», eMarketer Digital Intelligence, The eMarketer Blog. New York: Investigación y análisis de medios, 11 marzo 2010, Publicación seriada mensual. Disponible en: http://www.emarketer.com/blog/index.php/mobilemarketing-app-strategy-food-brand-consumer-product/ Visitado el 12 de octubre 2010 


\begin{tabular}{llrrr|}
\hline APP analizada & Categoría & $\begin{array}{c}\text { Valoración } \\
\text { estrellas* }\end{array}$ & $\begin{array}{l}\text { Opiniones } \\
\text { Usuarios }\end{array}$ & $\begin{array}{l}\text { Valoración } \\
\text { General** }\end{array}$ \\
\hline IKEA & HOGAR & 2,5 & 35 & 1 \\
\hline LA MAQUINISTA & OTROS & 2,5 & 6 & 1 \\
ZARA & MODA & 2 & 49 & 2 \\
MASSIMO DUTTI & MODA & 2 & 100 & 1 \\
CINESA & OTROS & 2 & 35 & 2 \\
ELLE & REVISTAS & 2 & 52 & 1 \\
MARIE CLAIRE & REVISTAS & 2 & 32 & 1 \\
GLAMOUR & REVISTAS & 2 & 20 & 1 \\
VOGUE STYLIST & REVISTAS & 2 & 20 & 2 \\
\hline IKEA INTERACTIVO & HOGAR & 1,5 & 4516 & 1 \\
\hline
\end{tabular}

* Valoración otorgada por los usuarios en función del uso, utilidad y éxito de la aplicación.

**Valoración 1=Deficiente, $2=$ Aceptable y 3=Óptima

Podemos encontrar cinco subdivisiones clasificadas en: Alimentación, Moda, Hogar, Revistas y Otros. A continuación describimos los resultados de cada subdivisión.

\subsection{Aplicaciones destinadas a la Moda}

Este es el tipo de aplicaciones que, junto con las revistas, cuentan con mayor representación. Corresponden a objetos o marcas de moda, específicamente relacionadas con marcas de ropa prêt-à-porter que se pueden conseguir de forma presencial en puntos de venta tales como Zara o Mango.

Las Marcas más destacadas según la valoración de los usuarios y refiriéndonos a la clasificación, sólo con catálogo, fueron Tous, $H \& M, D \& G$, Gucci y Massimo Dutti.

En estas aplicaciones observamos una gran variedad de formas en que las marcas se hacen presentes, y que van desde la presentación del catálogo de temporada, hasta la posibilidad de compra on line, esta opción solo se da en el caso de Zara y Mango. El resto de aplicaciones se limitan a mostrar sus colecciones con detalles, en algunos casos se incluyen las tiendas más cercanas y los precios como en el caso de H\&M, Blanco, Gucci y Massimo Dutti. Otro caso, como Tous, convierte el catálogo en un divertido juego que permite conocer la colección de una forma más amena. Es $D \& G$ quien gestiona esta aplicación de una forma más completa, permitiendo estar al tanto de sus noticias, desfiles y colecciones, además de contar con vínculos externos en páginas Web para ampliar la noticia destacada.

Algunas de estas marcas de moda, proporcionan incluso la opción de obtener mayor información y formar de parte de su grupo en Facebook y Twitter, vinculando su aplicación a estas redes sociales, lo que permite a la marca llegar a sus targets como un ente social.

La calificación numérica que asignan su usuarios en estrellas se mantiene como media en 2,5, la mejor valorada en este sentido es la página de Tous, que además del 
catálogo, presenta una serie de explicaciones de la aplicación como un juego, presenta la marca como algo divertido, en el caso de esta colección específicamente vinculado al mundo de la magia de la mano de Kylie Minogue (Figura 3). Esta marca cumple uno de los criterios en que Noha Elkin hace hincapié, y es el hecho de que la experiencia del usuario en las aplicaciones móviles debe ser complementaria y coherente con su experiencia a través de otros canales (léase punto de venta, página web, etc). En el caso de Tous se aplica, la marca no se limita a trasladar su catálogo a la app, sino que dentro de la aplicación crea una experiencia complementaria para el usuario, coherente siempre con la identidad de su empresa.

Fig. 3. Interfaz app TOUS
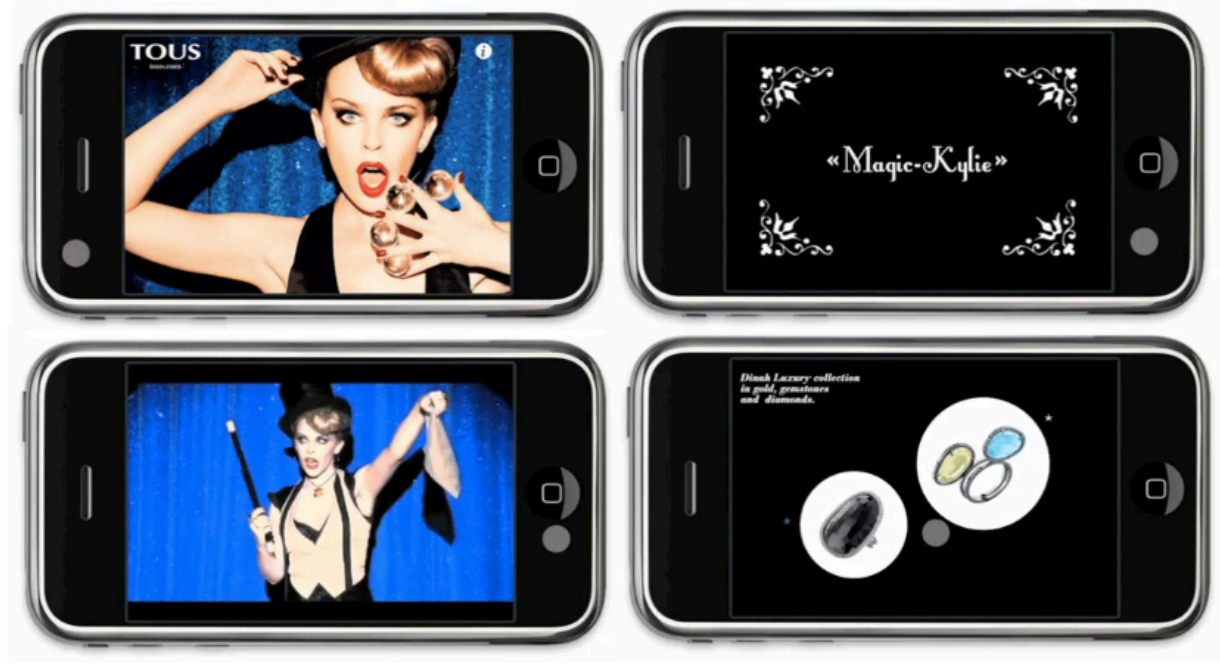

La queja principal en el caso de las aplicaciones en las que no se puede acceder a la compra de producto vía app, es precisamente la carencia de un espacio de $e$ commerce, en la mayoría de ejemplos revisados, es necesario salir de la aplicación y dirigirse a la página Web para hacer la compra, lo que obliga al usuario a desarrollar una serie de pasos extras que ralentizan el proceso de compra. Nielsen (2009) menciona que los usuarios de móviles enfrentan varios obstáculos que superar; las pequeñas pantallas, el ancho de banda/descarga, problemas de tiempo y lo peor de todo, un sitio mal diseñado.

Dentro de este grupo, cabe destacar que la mayoría de aplicaciones solicita al usuario seleccionar el país en el que se encuentra. Esto permite por un lado mostrar la internacionalidad de la marca y por otro, supone el marco adecuado para permitir el acercamiento de los servicios de localización. Gracias a la posibilidad de transmisión de posición en tiempo real, la marca da a conocer cuáles son las tiendas más cercanas al usuario y facilitárselas. Cuando la aplicación no proporciona un espacio para la compra on line, el servicio de localización se valora positivamente. 


\subsection{Aplicaciones para Revistas}

En este sentido encontramos que este tipo de aplicaciones ocupa el segundo puesto en el ranking de la muestra. Prácticamente todas las aplicaciones de revistas muestran en su inicio una síntesis de los artículos y espacios de la revista física pero no el artículo completo o si lo hacen, son pequeñas notas o recomendaciones. Parece ser que la forma de gestionar las revistas en todos los casos es la de presentar un menú de artículos a los que el usuario llega por elección, para encontrarse con la recomendación específica de un producto o marca. En el caso de estas aplicaciones, prácticamente en todas aparece una banderola en la parte baja que recomienda a otras revistas del mismo grupo. La sensación de este tipo de aplicación es que permite a la marca la venta de sus espacios por otras marcas y así llegar a su consumidor final solo como la herramienta que es a nivel físico, es decir, un espacio que gestiona al mismo tiempo artículos de interés y la venta de pequeños espacios a otras marcas, mostrando a la aplicación como un escaparate para la recomendación de productos vinculados al target.

Son pocas las opiniones que ven a esta aplicación como un camino para obligar al usuario a la compra física de la revista o a la suscripción de la misma, de hecho las opiniones han valorado la aplicación en su funcionamiento, sin embargo se observa una participación reducida en las opiniones, salvo en el caso de Cosmopolitan, que presenta una considerable diferencia en el número de valoraciones, con un total de 579.

La nota de valoraciones en este caso se vuelve a situar como media en el 2,5 estrellas, encontrando una gran variedad de opiniones que van desde el describir a la aplicación como genial y que permite estar al tanto de informaciones generales vinculadas al mundo de la moda, como que es una aplicación que no es actualizada constantemente lo que deteriora la imagen de marca. En este sentido es importante hacer énfasis en el hecho de que este tipo de aplicaciones requiere por parte de la marca no solo presencia, sino una correcta gestión de la aplicación, pues su mal uso, desarrollo técnico, e incluso su carencia de actualizaciones puede ir en detrimento de la marca.

Una única excepción a todas las revistas en aplicaciones analizadas es ELLE, que además de presentar la aplicación de la revista bajo los criterios presenta una aplicación vinculada: ELLE Gourmet (Figura 4), en cobranding con el supermercado de El Corte Inglés, una aplicación que presenta una amplia variedad de recetas, incluyendo la propuesta de receta del día, de fácil preparación y con todos los ingredientes, además de ofrecer la posibilidad de elegir receta por tipo de producto, de evento o de calorías. Una aplicación con una nota por encima de la media en este tipo de aplicaciones, 4 estrellas sobre 5 , catalogada como excelente y con una gran variedad de posibilidades. 
Fig. 4. Interfaz app ELLE Gourmet

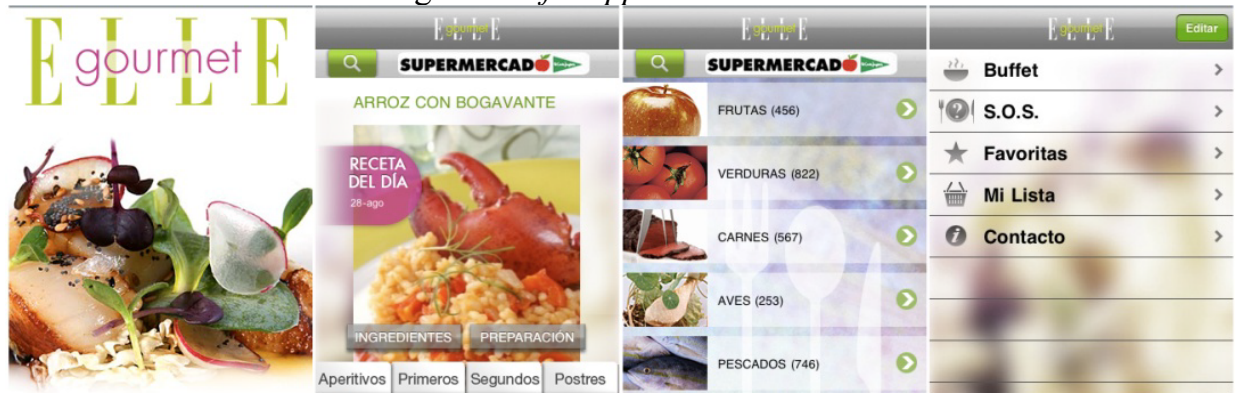

\subsection{Aplicaciones destinadas a Alimentación}

Las apps destinadas a la alimentación, ocupan un tercer puesto en las aplicaciones observadas. En este caso corresponden a marcas variadas, como son Nespresso, Telepizza y Philadelphia. En los dos primeros casos derivan en la venta de sus productos, ya que cuentan con servicio de e-commerce, lo que se convierte en un facilitador más, un sustituto tanto de Internet, como de la compra en punto de venta. Este tipo de herramientas, facilita al comprador el acceso al producto a distancia y permite conocer las actuales promociones del producto ofertado. La media de valoración de las 5 estrellas destinadas por Apple a este tipo de aplicaciones, en este caso se queda en 2.5, con opiniones variadas. En algunos casos se habla de la aplicación como estafa y en otros, se habla de la falta de un desarrollo técnico acorde al modelo de teléfono con que cuenta el usuario.

En lo concerniente a la aplicación de Philadelphia, se muestra una preocupación por la marca, no solo de ofertar un producto, sino de permitir al usuario la variedad en el consumo del mismo, ya que lo que presenta en su aplicación es un recetario que permite la grabación de recetas, el acceso a una lista de compra, etc. En todo caso no es más que una herramienta de apoyo que si formara parte de la Web de la marca solo sería un apartado más, pero en el caso de las aplicaciones, se convierte en la representación de la marca en este entorno. Podemos, entonces, distinguir en este tipo de aplicaciones, dos variantes que se repetirán a lo largo del análisis del resto de marcas de este estudio, es decir, el primero de los casos convierte a la aplicación en una pequeña Web vinculada al producto o servicio, que intenta ofrecer en la misma, algunos servicios vinculados a la Web, como son el e-commerce y el acceso a promociones, la segunda variante se corresponde a una manifestación de la marca, una intención de estar presente en esta tecnología, pero como una herramienta de la vida diaria del usuario. 


\subsection{Aplicaciones relacionadas con Hogar}

Este tipo de aplicaciones ocupan el cuarto puesto, y encontramos solo dos marcas que ha desarrollado aplicaciones: Zara Home e IKEA. En el caso de Zara Home, esta aplicación cuenta con un uso básico, ya que se limita a llevar al usuario a su página Web.

IKEA por su parte, presenta dos aplicaciones diferentes; la primera es el catálogo, una reproducción fiel del impreso y que permite no tener a mano la pieza física pero no aporta nada más como un valor añadido. Se ha intentado desarrollar en la segunda de sus aplicaciones un catálogo interactivo. Una opción más parecida a la realidad aumentada que permite hacer una foto de tu espacio físico incorporando algún elemento del catálogo IKEA, una aplicación divertida que permite, a aquellas personas que no tienen la facilidad de imaginar, justificar su elección.

Sin embargo, ambas apps presentan graves carencias con respecto al entorno en el que se desarrollan. Hay que destacar que el catálogo interactivo ha tenido la mayor cantidad de valoraciones de todas las aplicaciones descargadas, un total de 4.516, sin embargo, las notas de valoración la sitúan por debajo de la media, con 2 estrellas sobre 5. Las opiniones vertidas por parte de los usuarios la clasifican como: inútil, decepcionante, malísima, ni siquiera sirve como un catálogo de consulta.

La marca IKEA, no ha tenido en cuenta uno de los criterios más importantes que considera Elkin como condicionantes de una correcta aplicación, y es que una rápida incorporación a un nuevo entorno, en este caso las apps, si no es estudiado correctamente y gestionado de acuerdo a los criterios de identidad de la marca y a una correcta estrategia de comunicación, puede ir en detrimento de su imagen.

\subsection{Aplicaciones sobre Otros}

Aunque su representación es mayor que en el caso de las de gastronomía su variedad es amplia, podemos encontrar bajo este concepto marcas de diversos productos y servicios. Podemos mencionar por ejemplo el caso de Zippo, conocida marca de mecheros cuya aplicación en iPhone (Figura 5), corresponde a un sencillo juego visual basado en el movimiento típico de un mechero al encenderlo, la única opción que vincula al juego del mechero con la marca es que precisamente el mechero en cuestión es un Zippo y si el usuario quiere variar la estética del mismo por otro de la nueva colección de la marca, es necesario pagar alrededor de 1 euro por modelo. A través de esta aplicación puedes conocer el catálogo de diseños del producto pero solo es posible comprar su versión virtual. Hay que destacar que, debido a que ésta es una aplicación que permite un juego visual, se descarga en parte no solo por la relación con la marca sino por la novedad del juego que presenta, como podría ser el juego de beber cerveza utilizando la sensibilidad del iPhone a la posición. Esta aplicación ha obtenido una valoración de 3 estrellas, considerando que es una de las más valoradas de las aplicaciones analizadas, con 3.035 valoraciones. 
Otra de estas aplicaciones clasificas bajo el concepto Otros, es el caso de eBay. La conocida tienda de subastas virtual, ha desarrollado su aplicación en iPhone de cara a facilitar el seguimiento de las pujas a los compradores habituales. Una aplicación práctica valorada muy favorablemente por los usuarios con una nota por encima de los 3,5 estrellas.

Fig. 5. Interfaz app Zippo
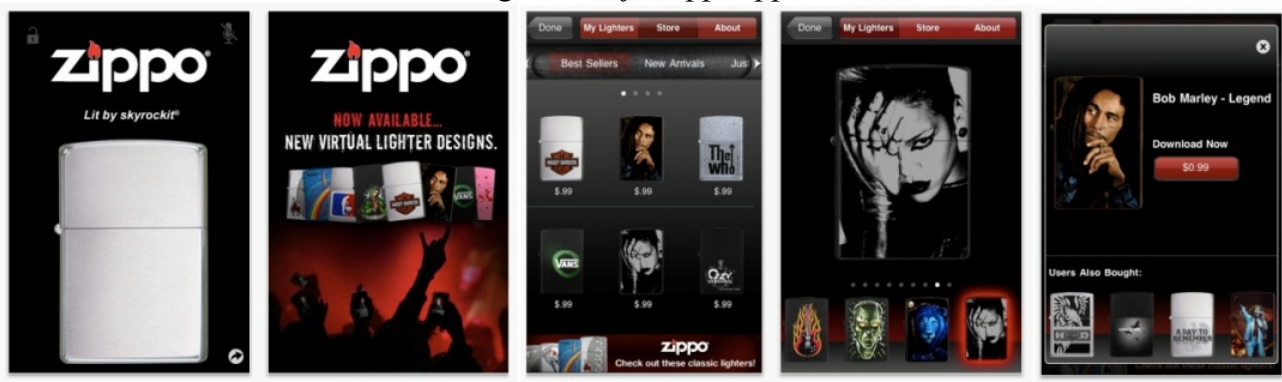

La Maquinista es otra de las aplicaciones vinculadas a una marca, y que corresponde a un centro comercial. Esta es una derivación de su página Web, donde se pueden encontrar las tiendas y novedades que se llevan a cabo en el mismo centro. No permite más allá de una clasificación de las tiendas que la conforman por tipología y solo proporciona datos como teléfono y número de local dentro del centro comercial. Consideramos que es de utilidad limitada para usuarios cercanos al centro, o asiduos del centro comercial. Aunque medianamente, es valorada por sus usuarios que en este caso participan con tan solo 6 valoraciones. La opinión expresada en el espacio se limita a un «por fin, un centro comercial en iPhone», pero no destaca las ventajas de la aplicación y por que considera necesaria la misma.

La última de estas marcas catalogadas, es Cinesa, una marca distribuidora de películas, y que muestra varios centros. La aplicación es en realidad un servicio que permite localizar de forma rápida las películas, sin necesidad de recurrir a la cartelera o a Internet. Permite a la marca dar un valor añadido y facilitar a los cinéfilos la localización de sus salas e incluso la compra directa de entradas. Obviamente, y casi como el resto de aplicaciones muestra observaciones son positivas y negativas a la marca. Podemos mencionar que algunos usuarios consideran esta app como un plagio de otra aplicación semejante desarrollada para otros cines.

\section{Conclusiones}

En este análisis, buscamos saber cómo las apps pueden favorecer o perjudicar a las marcas, y hemos visto que para el desarrollo de una aplicación de forma acorde a la identidad y gestión de la marca es indispensable tener en cuenta tres criterios básicos; el los anunciantes deben centrarse en el análisis de las necesidades de los 
usuarios, determinar sus objetivos, elaborar planes de integración y sobre todo, preparar medios de comunicación multicanal y de apoyo a la comercialización, pues si la aplicación no tiene un objetivo claro para el usuario, se percibirá como inútil, deteriorando la imagen de la marca de cara al cliente. Esto se refleja con el caso de una app de venta on line, si la aplicación funciona de forma adecuada, pero la cuestión logística no responde a los estándares de calidad ni de la marca ni de la aplicación, proporciona como consecuencia un deterioro de la imagen de marca debido a que daña la percepción del usuario/comprador.

En segundo lugar, el elemento esencial del proceso de construcción de las marcas que viven y se desarrollan en la web es la interactividad (Scolari, 2008), por tanto la experiencia del usuario en las apps debe ser complementaria y coherente con su experiencia a través de otros canales, es decir, debe mantenerse fiel a la marca y a la identidad de la empresa. Por último, es importante para la marca adaptarse constantemente a las nuevas tecnologías, pero no debemos olvidar que una incorporación precaria y mal desarrollada puede ser más perjudicial que beneficiosa.

Teniendo en cuenta esta información y los resultados mostrados con anterioridad pudimos observar que la aplicación mejor valorada por los usuarios es la de ELLE Gourmet. Precisamente, es una aplicación vinculada a la revista que consigue posicionarse muy por encima de la aplicación de la propia revista. Ya comentamos en su momento que es la única revista que ofrece un valor añadido, y es precisamente por ese valor adicional que da al usuario por lo que es valorada. Algunos de los comentarios por parte de los usuarios, y que podemos destacar son: «Las recetas que presentan son fáciles y muy bien explicadas», «hay una gran variedad de opciones», «Es gratuita», «Gracias a esto puedo comer mejor». Por otro lado, hay aportaciones que destacan aspectos negativos de la app, el más común es que la aplicación en ocasiones presenta problemas técnicos: «se cuelga al abrirla». En la mayoría de estos casos este defecto tiene que ver con el hecho de la utilización de una versión no actualizada.

En general es posible dar un valor añadido a la marca ELLE, incluso con herramientas que poco tienen que ver con el producto en sí. La app se vincula a su identidad y sobre todo a la visión sobre su gestión de marca, y el desarrollo de esta favorece la visión positiva por parte de los públicos. Esto sucede por ejemplo en el caso específico de $E L L E$, que mantiene, para esta aplicación, una colaboración con el supermercado de El Corte Inglés. La aplicación mantiene los mimos estándares de calidad que las revistas de recetas Apetece (revista física del supermercado de $E l$ Corte Inglés que se envía a domicilio).

En segunda posición dentro del ranking de las apps mejor valoradas, encontramos a Vogue, Tous, eBay y Cosmopolitan.

En el primer caso, Vogue mantiene estéticamente la línea de las otras revistas, denotando una mejor posición social con respecto al target. Al ingresar a la aplicación y como si se tratase de una revista física, podemos observar un anuncio; un spot de un perfume reproducido sin problemas técnicos ni de carga y con gran cali- 
dad de imagen. Algunas de las valoraciones hablan de la facilidad de navegación y la comodidad en su utilización, así como de una gran calidad en las imágenes. Destacan la presentación de un amplio catálogo de tendencias y pasarelas de moda, así como el hecho de contar con videos de alta calidad. Hay quien incluso recomienda «otras revistas deben tomar nota de la forma en cómo Vogue lleva su aplicación». Un punto a favor de la marca, que muestra la posibilidad de trasladar una identidad de marca a una aplicación que consigue reforzar la imagen de la misma frente al consumidor.

Tous ha conseguido trasladar la excelencia de su marca y comunicación a su aplicación, mediante una app bien desarrollada a nivel gráfico, que consigue ser una entidad en si misma, sin desvincularse de los criterios de comunicación de la marca. Las usuarias, generalmente mujeres, demuestran su agrado y hablan de: «una aplicación genial, perfecta, original y divertida» «estamos encantadas». Tous representa por tanto, otro ejemplo de una correcta utilización de esta herramienta de comunicación con los consumidores finales.

$e B a y$ es un caso un poco diferente pues aunque es una marca, es también una herramienta a través de la cual los usuarios pueden vender y subastar objetos, y ha conseguido posicionarse como un espacio de subasta referente en su sector. Al parecer ha conseguido trasladar esa imagen a la aplicación. La gran ventaja en este sentido es que la aplicación funciona correctamente, tanto funcionalmente como en transmitir sus valores, aunque al final la app no es más que la reproducción del espacio web en una aplicación. eBay como partner de Netbiscuits, un proveedor de software que permite que la aplicación funcione correctamente, proporciona una convincente experiencia móvil para todos los usuarios de teléfonos inteligentes ${ }^{14}$. Y al parecer así es, puesto que la mayoría de los comentarios mencionan sobre todo la facilidad que da este tipo de aplicación de cara a los seguimientos en subastas a tiempo real, pues permite al usuario prescindir del uso del ordenador para lleva a cabo una puja. Una aplicación que completa de forma muy coherente su imagen, y que ha sido gestionada técnicamente de tal forma que se adapta a las características y habilidades del usuario. En general, es una aplicación muy valorada por que facilita al usuario el acceso y elimina el gasto del tiempo de conexión en un ordenador fijo.

La última app destacada con mejor valoración es Cosmopolitan. Curiosamente aparece como aplicación dos veces en la misma búsqueda sin que quede muy clara la diferencia entre una y otra app prácticamente iguales aunque diferenciadas en que a una la llaman 2.0 y a la otra simplemente Cosmopolitan. Destacable el hecho de que es precisamente la que no es 2.0 , la que presenta una valoración considerablemente mejor. La mayoría de los comentarios positivos en esta aplicación giran en torno a «está actualizada, con contenidos divertidos e interesantes», «tiene un diseño cuidado» «la aplicación es gratuita». El consumidor la percibe como un

14 KATS, Rimma, «La Redoute lets consumers snap and buy items directly from print catalog. Mobile Commerce Daily. Publicación seriada semanal. Disponible en: http://www.mobilecommercedaily.com/ebayimproves-mobile-site-for-efficient-consumer-buying-and-selling/ Visitado el 16 de octubre 2010. 
regalo de la revista, que aunque muestra artículos cortos e información sintetizada, es muy útil para las fashionistas. Como puntos desfavorables podemos mencionar que en algunos casos las opiniones se quejan de que los contenidos no se actualizan semanalmente.

Con diferencia, una de las aplicaciones peor valoradas y con mayor número de participaciones, 4.516 en total, es la del catálogo virtual de IKEA, una aplicación ampliamente desarrollada técnicamente, pero sin embargo, que no proporciona un valor añadido a la marca, sino al contrario, ha conseguido deteriorar la imagen de marca. A través de esta aplicación es posible colocar los muebles de IKEA en un espacio real y visualizar como se verían, los graves problemas que se detectan a partir de las opiniones son que no se cuenta con un catálogo completo de los productos ni de precios, una aplicación considerada como inservible y que no se relaciona en absoluto con la descarga del catálogo, otra aplicación también desarrollada por la marca. Importante tener en cuenta una de las observaciones que menciona literalmente: «Por Dios, y a saber lo que habrán pagado por esto. »

En este sentido es importante hacer énfasis en el hecho de que este tipo de aplicaciones requiere por parte de la marca no solo presencia, sino un claro objetivo y una correcta gestión de la aplicación, pues su mal uso, desarrollo técnico, e incluso su carencia de actualizaciones puede ir en detrimento de la marca.

Como conclusiones generales, en base a los resultados obtenidos, podemos mencionar que para que una aplicación sea valorada correctamente, y por ende, la marca sea percibida por sus públicos de una forma positiva, hay algunos factores a tener en cuenta: Jami Lawrence, director asociado de Publicis Modem \& Dialog menciona que es imprescindible que las aplicaciones proporcionen al usuario cualquiera de estas dos experiencias: diversión o ahorro de tiempo, y hemos observado que son precisamente ambas características las que son destacadas en las aplicaciones mejor valoradas, Tous en el caso de la diversión y eBay en el caso del ahorro de tiempo. Hemos de añadir que algunos de los factores relacionados con la correcta percepción de la marca también tienen que ver con el hecho de que la marca proporciona un valor añadido, como es el caso de ELLE Gourmet, o de que la aplicación presente una gran calidad de imágenes y sea cómoda y fácil de utilizar.

Otro de los factores que se valoran en las aplicaciones, según Jami Lawrence es el hecho de que la aplicación tenga sentido para el usuario y tener un valor añadido que no necesariamente tiene que estar relacionado con la naturaleza de la marca, aunque siempre, cualquier aplicación a desarrollar ha de ser coherente con la identidad de la marca.

Es necesario, para concluir, añadir que este estudio no intenta presentar los valores necesarios para que la marca sea gestionada correctamente en las aplicaciones, pretende solo ser una aproximación a la realidad actual del mercado e intentar marcar algunas pautas para la correcta gestión en la identidad de las marcas de cara a la mejor percepción de la marca por parte sus públicos. Lo que lleva a la conclusión de que así como no hay un dispositivo para todos, ninguna plataforma es la 
adecuada para todas las marcas si no forma parte de una correcta estrategia de gestión de la misma.

\section{Referencias bibliográficas}

AGUADO, Juan Miguel y MARTíNEZ, Inmaculada (2009): «Mobile Media Implicit Cultures: Towards a Characterization of Mobile Entertainment and Advertising in Digital Convergence Landscape», Observatorio Journal, 13, 336-352

ARRANZ, Juan Carlos (1997): Gestión de la Identidad Empresarial y su impacto sobre los resultados. Barcelona, Ediciones Gestión 2000, S. A.

BANTZ, Charles (1993): Understanding Organizations. Interpreting Organizational Communication Cultures. Columbia, University of South Carolina Press

BERRY, Leonard (2004): Un buen Servicio ya no basta. Más allá de la excelencia en el servicio. Bilbao, Ediciones Deusto.

DANS, Enrique (2010): Todo va a cambiar. Tecnología y evolución: adaptarse o desaparecer, Barcelona, Grupo Planeta.

DistiMO Report (2011): The battle for the most content and the emerging tablet market. Distimo Publication, April 2011, Netherlands

CANAlys (2010): Expert Analysis for High-tech Industry: Global Smart Phone Shipments Rise 28\%. Reading. UK, 2008. Disponible en: http://www.canalys.com/pr/2008/r2008112.html Visitado el 10 de octubre 2010

CAPRIOTTI, Paul (2009): Branding Corporativo. Fundamentos para la gestión estratégica de la identidad Corporativa, Santiago de Chile, Colección Libros de Empresa, Andros Impresores.

CORBETA, Piergiorgio (2007): Metodología y Técnicas de investigación social, Mc Graw Hill,

ElKIN, Noha (2010): «Mobile Marketing Trends, Insights and Best Practices». eMarketer Digital Intelligence.The eMarketer Blog. New York: Investigación y análisis de medios, 26 agosto 2010, Publicación seriada mensual. Disponible en:

http://www.emarketer.com/blog/index.php/emarketer-webinar-mobile-marketingtrends-insights-practices/Visitado el 12 de octubre 2010

FeiJoo, Claudio y MAghiRos, Ioannis (2008): Mobile content. On the verge of an explosion. JRC Scientific Reports. Sevilla: EC. IPTS.

FERNÁNDEZ NOGALES, Ángel (2010): «La situación actual de las marcas de distribuidor desde la perspectiva de los fabricantes: un estudio cualitativo», Distribución y Consumo, 113, 24-35

KATS, Rimma (2010): La Redoute lets consumers snap and buy items directly from print catalog. Mobile Commerce Daily. Publicación seriada semanal. Disponible en: $\mathrm{http} / / / \mathrm{www}$.mobilecommercedaily.com/ebay-improves-mobile-site-for-efficientconsumer-buying-and-selling/l Visitado el 16 de octubre 2010

LaWrence, Jami (2010): «Best Practices: Mobile Marketing \& App Strategies for Food Brands», eMarketer Digital Intelligence, The eMarketer Blog. New York: Investigación y análisis de medios, 11 marzo 2010, Publicación seriada mensual. Disponible en: http://www.emarketer.com/blog/index.php/mobile-marketing-app-strategy-food-brandconsumer-product/ Visitado el 12 de octubre 2010 
NIELSEN, Jakob (2011): «Mobile Usability» Jakob Nielsen's Alertbox, 20 de Julio 2009. Disponible en: http://www.useit.com/alertbox/mobile-usability.html Visitado el 1 de junio de 2011

SANZ De LA TAJADA. Luis Ángel (1994): Integración de la Identidad y la imagen de la empresa. Desarrollo conceptual y aplicación práctica, Madrid, ESIC editorial.

SCOLARI, Carlos (2008): «Online brands: Branding, possible worlds, and interactive grammars» Semiotica, 169-178

The NiElsen COMPANY (2011): «The State Of Mobile Apps» Nielsen Wire, Nielsen.com, Junio de 2010. Disponible en: http://blog.nielsen.com/nielsenwire/online_mobile/thestate-of-mobile-apps/ Visitado el 11 de mayo de 2011

Villafañe, Justo (1993): , Imagen Positiva, Gestión Estratégica de la Imagen de las Empresas, Madrid, Ediciones Pirámide.

VACAS AGUILAR, Francisco (2007): «Telefonía Móvil: la cuarta ventana», ZER, 23, 199217

Recibido: 4 de abril de 2011

Aceptado: 16 de diciembre 2011 